\section{O Programa de Humanização no Pré-natal e Nascimento do Ministério da Saúde no Brasil: resultados iniciais}

\author{
The Brazilian Ministry of Health's Program \\ for Humanization of Prenatal and Childbirth \\ Care: preliminary results
}

Suzanne Jacob Serruya 1 José Guilherme Cecatti 2 Tania di Giacomo do Lago 3
${ }^{1}$ Departamento de Ciência e Tecnologia, Ministério da Saúde, Brasília, Brasil. 2 Departamento de Tocoginecologia, Universidade Estadual de Campinas, Campinas, Brasil.

3 Faculdade de Ciências Médicas, Santa Casa de São Paulo, São Paulo, Brasil.

Correspondência S. J. Serruya Departamento de Ciência e Tecnologia, Ministério da Saúde. Esplanada dos Ministérios, Bloco G, Ed. Sede, 8 o andar, Brasília, DF 70058-900, Brasil. suzanne.jacob@saude.gov.br

\section{Abstract}

This article evaluates the implementation of the Brazilian Ministry of Health's Program for Humanization of Prenatal and Childbirth Care us ing data generated by the SISPRENATAL/DATASUS database from the Unified National Health System. From its beginning in June 2000 until December 2002, 3,983 municipalities joined the Program, and $71 \%$ of participating municipalities $(3,183)$ reported their health care activities, constituting a database with 720,871 women. Nearly $20 \%$ of the women had six or more prenatal visits, and approximately half of them had the postpartum follow-up visit and required lab tests performed in 2002. In addition, $41 \%$ of the women had been vaccinated against tetanus. The number of HIV antibody tests was twice that of syphilis during the two-year period. Only a small percentage of women (2\% in 2001 and 5\% in 2002) received the entire set of prenatal and childbirth care services. The low percentages attest to the need for permanent evaluation aimed at improving quality of care and guaranteeing both high-quality maternal and perinatal results and the inalienable right of women to safe care and well-being during pregnancy and delivery.

Maternal and Child Health; Women's Health; Program Evaluation

\section{Introdução}

Melhorar a saúde materna e impedir mortes evitáveis é, ainda, um dos objetivos de maior interesse nacional e internacional no campo da saúde e dos direitos reprodutivos, no qual se discutem quais as medidas necessárias e eficazes para alcançar tal propósito 1,2,3,4,5,6. Entretanto, é necessário conjugar a segurança de obter bons resultados com o bem-estar para a mulher e o recém-nascido, respeitando-se direitos constituídos 7,8,9,10.

No Brasil, a atenção à mulher na gestação e parto permanece como um desafio para a assistência, tanto no que se refere à qualidade propriamente dita, quanto aos princípios filosóficos do cuidado, ainda centrado em um modelo medicalizante, hospitalocêntrico e tecnocrático 11 .

A assistência à gestante, uma das atividades realizadas há mais tempo nos serviços públicos de saúde no país, foi, por muitos anos, orientada principalmente para melhorar os indicadores da saúde infantil. Um novo paradigma na atenção à saúde da mulher foi concebido pelo movimento de mulheres em associação com profissionais de saúde e traduzido nas bases programáticas do Programa de Assistência Integral à Saúde da Mulher (PAISM), instituído pelo Ministério da Saúde (MS) em 1983 12,13. A partir da 8a Conferência Nacional de Saúde, em 1986, e da promulgação da Constituição, em 
1988, o direito à saúde estaria garantido por lei e um sistema único de saúde deveria ser implantado de forma descentralizada e com instâncias de controle social 14.

As bases filosóficas do PAISM introduziam outros conceitos na atenção à saúde feminina, como a integralidade e a autonomia corporal, que deveriam ser estimuladas e discutidas nas ações educativas articuladas ao programa. A partir de critérios epidemiológicos que incorporavam a especificidade da condição feminina como determinante do processo saúde-doença, os profissionais de saúde deveriam ser capacitados para incorporarem essa visão e estabelecerem novas práticas (PAISM-1984). Do ponto de vista conceitual, a operacionalização da idéia de integralidade foi o maior desafio, uma vez que exigia dos gestores e profissionais uma postura que extrapolava a capacitação técnica para responder isoladamente a cada um dos problemas femininos de saúde. Essa nova atitude requeria que os profissionais se relacionassem com as mulheres enquanto sujeitos e detentoras de direitos, sendo compreendidas suas demandas reprodutivas e relativas à sexualidade no contexto de subordinação das mulheres nos planos privado e público.

Desse modo, o PAISM apresentou um deslocamento de olhar na atenção à saúde da mulher, obrigando os serviços e gestores a pensarem de forma mais ampla sobre a questão. Nestas quase duas décadas já passadas, a implantação do PAISM foi bastante diferenciada em todo o país, refletindo os diferentes graus de compromisso político dos governantes com a questão da mulher, a heterogeneidade nas estratégias adotadas para o desenvolvimento e a organização do sistema de saúde. Alguns estudos abordaram os diversos aspectos do programa, enfatizando as questões da saúde reprodutiva, a introdução de conceitos como a integralidade, os desafios ligados à sua implementação, enfim, as diferentes questões que permanecem atuais até o momento 12,13,15.

Observa-se que, mesmo nos serviços que realizam o conjunto das atividades como preconizadas pelo PAISM, há questionamentos sobre a qualidade da assistência prestada e o impacto nos indicadores de resultados. Apesar da assistência pré-natal ter estado sempre presente no escopo das ações praticadas pelos serviços de saúde, até o momento, permanecem questões que, entre outras, devem ser discutidas, como o acesso em algumas regiões e áreas do país, a qualidade da atenção prestada, e o vínculo entre o pré-natal e o parto, a humanização da atenção e inaceitáveis taxas de mortalidade materna e perinatal (Programa de Hu- manização no Pré-natal e Nascimento; Secretaria de Políticas de Saúde, Ministério da Saúde. Urgências e Emergências Maternas. Guia para Diagnóstico e Conduta em Situações de Risco de Morte Materna).

Entendendo que a não percepção da mulher como sujeito e o desconhecimento e desrespeito aos direitos reprodutivos constituem o pano de fundo da má assistência, o MS instituiu, em junho de 2000, o Programa de Humanização no Pré-natal e Nascimento (PHPN) no qual o respeito a esses direitos e a perspectiva da humanização aparecem como elementos estruturadores.

A fundamentação da medida ministerial, na instituição das portarias do PHPN, pretende ser um marcador de águas anunciando o paradigma da humanização como novo modelo de atenção à mulher durante a gestação e o parto. Desse modo, na cartilha de apresentação do PHPN, a humanização da assistência é ratificada como a principal estratégia, seguida da apresentação dos aspectos conceituais sobre humanização: “a principal estratégia do Programa de Humanização no Pré-natal e Nascimento é assegurar a melhoria do acesso, da cobertura e da qualidade do acompanhamento pré-natal, da assistência ao parto e puerpério às gestantes e ao recém-nascido, na perspectiva dos direitos de cidadania. O Programa fundamenta-se no direito à humanização da assistência obstétrica e neonatal como condição primeira para o adequado acompanhamento do parto $e$ do puerpério. A humanização compreende, entre outros, dois aspectos fundamentais. O primeiro diz respeito à convicção de que é dever das unidades de saúde receber com dignidade a mulher, seus familiares e o recém-nascido. Isto requer atitude ética e solidária por parte dos profissionais de saúde, organização da instituição de modo a criar um ambiente acolhedor $e$ adotar condutas hospitalares que rompam com o tradicional isolamento imposto à mulher. $O$ segundo refere-se à adoção de medidas e procedimentos sabidamente benéficos para o acompanhamento do parto e do nascimento, evitando práticas intervencionistas desnecessárias que, embora tradicionalmente realizadas, não beneficiam a mulher nem o recém-nascido e que, com freqüência, acarretam maiores riscos para ambos".

No plano operacional, o PHPN definiu elementos chaves da assistência à gestação e ao parto, em torno dos quais deveria concentrar esforços a fim de alcançar o objetivo principal de reduzir as altas taxas de morbi-mortalidade materna e perinatal. Incluem-se aí a necessidade de ampliar o acesso ao pré-natal, o esta- 
belecimento de procedimentos e ações, cuja realização é fundamental para esse acompanhamento, e a promoção do vínculo entre a assistência ambulatorial e o momento do parto.

O PHPN instituiu ainda uma estratégia para induzir e auxiliar a maioria dos municípios a implementar essas ações, introduzindo novos recursos para o custeio dessa assistência e transferindo-os mediante o cumprimento de critérios mínimos, necessários para melhorar a qualidade da assistência. Os critérios recomendados foram: realizar a primeira consulta de prénatal até o quarto mês de gestação; garantir a realização dos seguintes procedimentos: no mínimo, seis consultas de pré-natal, sendo, preferencialmente, uma no primeiro trimestre, duas no segundo trimestre e três no terceiro trimestre da gestação; uma consulta no puerpério, até 42 dias após o nascimento; exames laboratoriais: (a) $\mathrm{ABO}-\mathrm{Rh}$, na primeira consulta; (b) VDRL, um exame na primeira consulta e outro próximo à trigésima semana da gestação; (c) Urina rotina, um exame na primeira consulta e outro próximo à trigésima semana da gestação; (d) Glicemia de jejum, um exame na primeira consulta e outro próximo à trigésima semana da gestação; (e) Hemoglobina/Hematócrito, na primeira consulta; oferta de testagem anti-HIV, com um exame na primeira consulta, naqueles municípios com população acima de cinqüenta mil habitantes; aplicação de vacina antitetânica até a dose imunizante (segunda) do esquema recomendado ou dose de reforço em mulheres já imunizadas.

Os incentivos financeiros foram assim estabelecidos: R\$ 10,00 no registro do cadastramento da gestante no PHPN e R\$ 40,00 por gestante na conclusão do pré-natal, desde que cumprido o elenco mínimo de procedimentos estabelecidos pelo PHPN.

A criação de um sistema informatizado de informação e acompanhamento do PHPN, o SISPRENATAL, foi considerada como medida fundamental do programa. Esse sistema de informações deveria, além disso, monitorar o pagamento dos incentivos financeiros e constituir-se em um instrumento capaz de fornecer um conjunto de relatórios e indicadores planejados para monitorar essa atenção em âmbito municipal e estadual, contribuindo para melhorar a gestão dos serviços. Até a instituição do PHPN, os dados referentes à assistência pré-natal no SUS restringiam-se ao número absoluto de consultas, não permitindo avaliações mais detalhadas da qualidade do cuidado pré-natal.

A avaliação proposta neste artigo refere-se à assistência pré-natal propriamente dita, com o objetivo principal de avaliar preliminarmen- te os indicadores gerados no PHPN do MS para o Brasil em dois anos (2001 e 2002).

\section{Método}

Foi realizado um estudo descritivo, inicialmente resgatando elementos do processo de elaboração e implantação do programa, e foi realizada uma avaliação dos dados gerados no SISPRENATAL nos dois primeiros anos do programa, 2001 e 2002.

Para a avaliação dos dados quantitativos, foram solicitadas ao Departamento de Informática do SUS (DATASUS) as planilhas de produção do país e dos estados dos períodos em estudo, bem como os indicadores do sistema já elaborados e incorporados no sistema de informação. Para as informações sobre adesão dos municípios, fornecidas pelo MS, foram feitas comparações simples entre os períodos estudados, bem como entre estados e regiões do país. O projeto deste estudo foi previamente aprovado pelo Comitê de Ética em Pesquisa da Faculdade de Ciências Médicas da Universidade Estadual de Campinas, e a utilização dos dados do banco DATASUS/SISPRENATAL foi autorizada pelo MS.

\section{Resultados}

A implantação do PHPN foi realizada formalmente com a adesão de 3.985 municípios até dezembro de 2002. A adesão ao PHPN, enquanto processo de organização da atenção obstétrica, foi realizada com mecanismos ainda imaturos de gestão, no que se refere à descentralização e à regionalização, gerando conflitos de atribuições nos três níveis de governo. A estratégia para a adesão proporcionou a discussão do panorama de atenção à gestação e ao parto em cada município, revelando fragilidades na capacidade instalada e na distribuição de recursos de média e alta complexidade. A implementação do programa e a instalação do sistema de informações, SISPRENATAL, com a produção de dados, ocorreram em 3.016 municípios, até dezembro de 2002. Foram cadastradas 720.871 mulheres nos dois anos em análise, 2001 e 2002 (Tabela 1). As Figuras 1 e 2 mostram a cobertura da adesão dos municípios ao PHPN no final de 2001 e 2002 respectivamente.

Segundo a análise dos indicadores do programa nesses dois anos (Tabela 2), a cobertura do programa mostra um incremento importante entre 2001 (9,25\%) e 2002 (27,92\%), com o cadastramento de 555.979 mulheres no segundo 
Histórico cronológico da apresentação de produção no Sistema de Informação Ambulatorial/SUS dos municípios com adesão e do número de gestantes cadastradas de outubro de 2000 a dezembro de 2002. SISPRENATAL, Brasil, 2000-2002.

\begin{tabular}{|c|c|c|c|c|}
\hline Mês/Ano & $\begin{array}{l}\text { Número de } \\
\text { municípios } \\
\text { cadastrando }\end{array}$ & $\begin{array}{l}\text { Número de } \\
\text { municípios } \\
\text { com conclusão }\end{array}$ & $\begin{array}{l}\text { Número de } \\
\text { gestantes } \\
\text { apresentado no } \\
\text { cadastramento }\end{array}$ & $\begin{array}{l}\text { Número de } \\
\text { gestantes } \\
\text { apresentando } \\
\text { conclusão }\end{array}$ \\
\hline Janeiro/2001 & 40 & 0 & 1.375 & 0 \\
\hline Fevereiro/2001 & 44 & 0 & 1.067 & 0 \\
\hline Março/2001 & 76 & 0 & 3.917 & 0 \\
\hline Abril/2001 & 87 & 0 & 3.685 & 0 \\
\hline Maio/2001 & 138 & 0 & 5.624 & 0 \\
\hline Junho/2001 & 425 & 2 & 9.532 & 3 \\
\hline Julho/2001 & 613 & 1 & 14.793 & 2 \\
\hline Agosto/2001 & 862 & 5 & 20.618 & 16 \\
\hline Setembro/2001 & 1.003 & 7 & 22.949 & 21 \\
\hline Outubro/2001 & 1.180 & 9 & 28.960 & 27 \\
\hline Novembro/2001 & 1.359 & 17 & 28.649 & 62 \\
\hline Dezembro/2001 & 1.439 & 48 & 23.723 & 101 \\
\hline Janeiro/2002 & 1.753 & 95 & 33.704 & 269 \\
\hline Fevereiro/2002 & 1.895 & 126 & 32.803 & 416 \\
\hline Março/2002 & 2.016 & 175 & 38.790 & 699 \\
\hline Abril/2002 & 2.167 & 222 & 44.874 & 1.042 \\
\hline Maio/2002 & 2.216 & 274 & 43.133 & 1.212 \\
\hline Junho/2002 & 2.263 & 327 & 41.155 & 1.485 \\
\hline Julho/2002 & 2.396 & 380 & 54.897 & 1.679 \\
\hline Agosto/2002 & 2.465 & 438 & 59.413 & 1.856 \\
\hline Setembro/2002 & 2.490 & 472 & 56.002 & 2.123 \\
\hline Outubro/2002 & 2.511 & 540 & 61.351 & 2.456 \\
\hline Novembro/2002 & 2.423 & 548 & 51.811 & 2.291 \\
\hline Dezembro/2002 & 2.228 & 551 & 38.046 & 2.225 \\
\hline
\end{tabular}

ano, a maioria inscrita com até 120 dias de gestação (cerca de 90\%). O percentual de mulheres que realizou seis consultas de pré-natal foi próximo de $20 \%$ nos dois anos estudados, e a conjugação desse critério com a consulta puerperal e/ou os exames obrigatórios reduziu esse percentual pela metade em 2002 . O registro da vacina antitetânica cresceu $100 \%$ de 2001 para 2002, enquanto o percentual de solicitações de exames para a detecção de sífilis foi a metade em relação a solicitações de exames para a AIDS nesses dois anos. Uma leitura vertical dos indicadores, nos dois anos em estudo, aponta que, à medida que se adicionam os critérios assistenciais, os percentuais diminuem, indicando que é a realização do conjunto das atividades o maior desafio na atenção pré-natal. Assim, apenas $2 \%$, em 2001, e 5\%, em 2002, das mulheres cadastradas realizaram o conjunto das atividades assistenciais estabelecidas.

\section{Discussão}

A elaboração do sistema de informações sobre o pré-natal disponibilizou relatórios e indicadores e desenhou um retrato da assistência preocupante e desafiador. Apesar dos baixos percentuais registrados, a comparação dos indicadores de qualidade de assistência, apontados pelo SISPRENATAL, revelou crescimento de 2001 para 2002. Entretanto, é permanente a necessidade de avaliações com o objetivo de melhorar a qualidade dessa atenção, garantindo melhores resultados maternos e perinatais.

A taxa de mortalidade materna configurase, entre os indicadores sócio-econômicos, como o índice que é capaz de melhor traduzir o status da condição feminina. Por esse motivo, a avaliação das estratégias usadas em diferentes países para diminuir a morbi-mortalidade materna e otimizar recursos é tema recorrente 
na análise da situação de saúde das mulheres ao longo do ciclo gravídico-puerperal 2,4,16,17, $18,19,20$.

Pesquisas qualitativas sobre assistência pré-natal indicam que a qualidade da assistência representa o nó crítico principal, mantendo-a como tema permanente de preocupação e discussão 21,22,23,24.

Por outro lado, os modelos de assistência pré-natal têm estado em profícuo debate 21,25 , 26,27 . Recente revisão sistemática avaliou comparativamente modelos de cuidado pré-natal, analisando um conjunto de variáveis e buscando definir um modelo que otimize recursos, utilize intervenções baseadas em evidências e que não adicione riscos às gestantes que não apresentam complicações 28 .

Na mesma direção, a Organização Mundial da Saúde publicou recentemente, com base em um ensaio clínico aleatorizado multicêntrico, os resultados de um novo modelo simplificado para a assistência pré-natal em gestantes de baixo risco, com resultados primários similares em termos de freqüência de pré-eclampsia, anemia severa pós-parto, infecção do trato urinário e baixo peso ao nascer, em relação ao modelo padrão ocidental de atenção pré-natal 29,30,31.

A discussão de propostas de assistência prénatal permanece atual e ainda é um campo fértil para estudos operacionais que incluam análises quantitativas e qualitativas 18,32. De fato, o PHPN permitiu retomar uma discussão sobre o pré-natal que parecia já superada. Embora ninguém discorde da importância do acompanhamento da gestação, e o número de consultas seja um constante indicador para avaliação e para o estabelecimento de pactos entre as diferentes esferas do governo, de fato, não existia definição clara e precisa sobre o que fazer. Na ausência de outros parâmetros objetivos, prevaleceram o número de consultas e a cobertura 33 (Indicadores da Assistência à Saúde, http: //www.saude.gov.br, acessado em Fev/2003).

Por outro lado, como a idéia, no senso comum, de "fazer pré-natal" é ter um certo número de consultas, mesmo a permanência de dados epidemiológicos negativos, como a alta taxa de sífilis congênita, não parecia sensibilizar a maioria dos gestores. Considerando que quase $90 \%$ das mulheres fazem pré-natal com profissionais de saúde formalmente qualificados, enfermeiros ou médicos, e a média de consultas, no SUS, está acima de quatro consultas por mulher, o Brasil já deveria apresentar melhores indicadores de saúde perinatal. Além disso, alguns estudos sobre a assistência materna apresentam outros claros sinais de descaso, como o número de mulheres com pron-
Figura 1

Municípios brasileiros aderidos ao Programa de Humanização

no Pré-natal e Nascimento até dezembro de 2001.

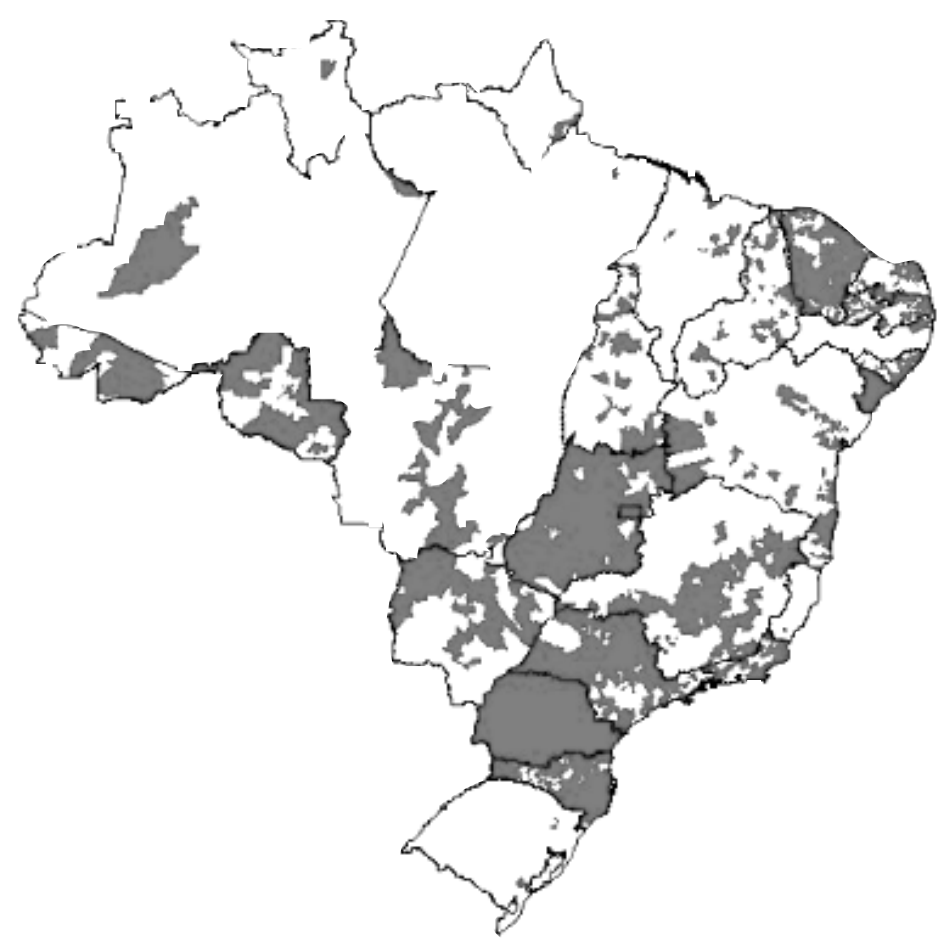

tuários sem anotações, cartões de acompanhamento não preenchidos e outros 22,32,34.

Um dos desdobramentos iniciais do PHPN foi recolocar a atenção pré-natal no cenário das demais intervenções voltadas para promover a saúde das mulheres, como estratégia necessária e de fundamental importância. A focalização nesse momento da atenção à mulher pode suscitar repercussões aparentemente contraditórias. Concentrar esforços e recursos para qualificar a ação mais antiga e tradicional de atenção à mulher para torná-la realmente efetiva é até uma forma de honrar um débito histórico. Por outro lado, pode parecer uma retomada da valorização exclusiva da mulher como "reprodutora". Como existem outras demandas não atendidas em relação à saúde da mulher, o PHPN pode ser interpretado como um atraso no atendimento dessas questões, mesmo que tivesse como objetivo a redução de mortes maternas. Essa contradição é, no entanto, aparente porque, em primeiro lugar, a proposta de melhoria da atenção obstétrica conduzi- 
Figura 2

Municípios brasileiros aderidos ao Programa de Humanização

no Pré-natal e Nascimento até dezembro de 2002.

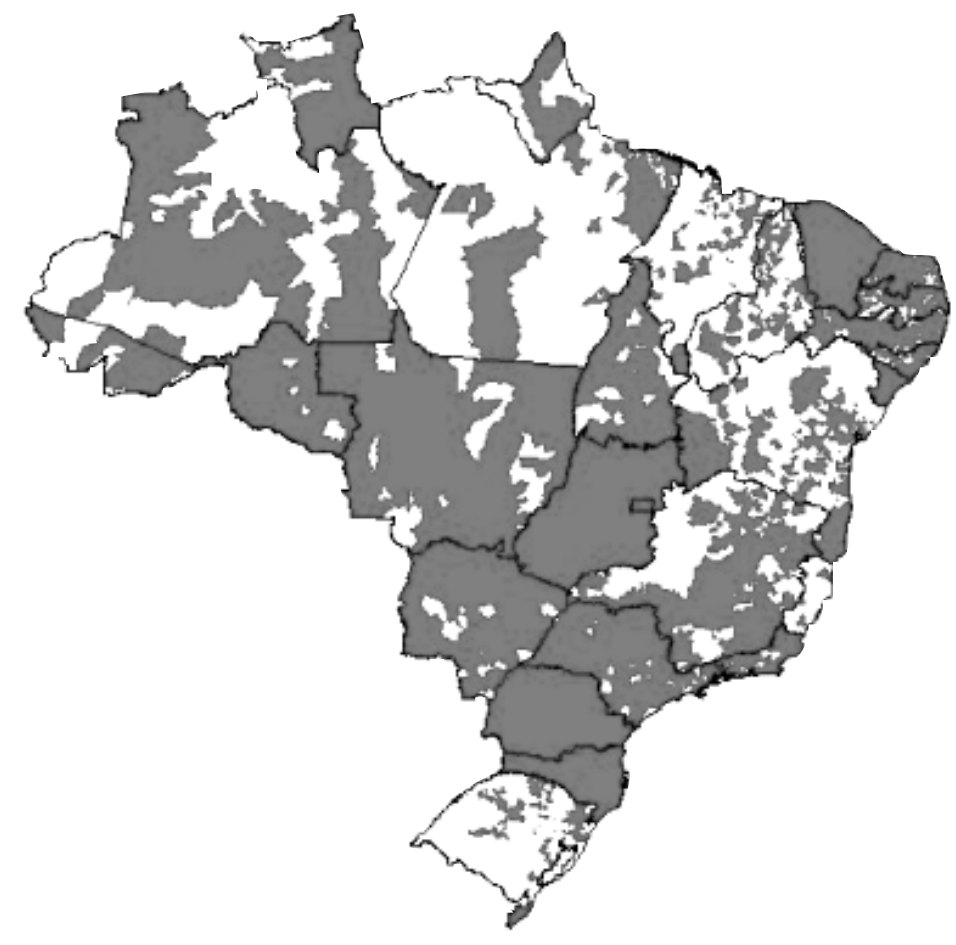

da pela humanização também é importante demanda da agenda de saúde das mulheres e, em segundo lugar, porque a focalização realizada na assistência com o PHPN parte de uma constatação praticamente consensual da necessidade de ampliar o acesso e garantir qualidade, e, por fim, a instituição do programa não teve como pressuposto ou conseqüência o não atendimento a outras demandas.

$\mathrm{O}$ incentivo financeiro do programa procurava privilegiar dois aspectos: a ampliação do acesso precoce traduzido no pagamento de $\mathrm{R} \$$ 10,00 na primeira consulta pela captação da gestante com até 120 dias de idade gestacional e a realização do conjunto de ações estabelecido com o pagamento do restante do incentivo ( $R \$ 40,00)$ na conclusão da assistência.

A análise do acesso, a partir dos dados disponíveis no SUS para o período de 2000 a 2002, mostra um incremento de $20 \%$, elevando a média de consultas por mulher que faz o parto no SUS de 3,4 em 1999 para 4,4 em 2002 (http:// www.saude.gov.br, acessado em Fev/2004).
Embora a manutenção do crescimento dessa razão seja um bom indicativo, não se pode afirmar que esse incremento seja devido ao programa. A questão do acesso, embora seja classicamente avaliada quantitativamente, merece um olhar qualitativo que possa perceber se o Programa incluiu justamente as mulheres que estão em franca desvantagem social, seja por localização, região, escolaridade, raça/etnia ou renda. Estudos de avaliação são necessários para complementar esta análise.

A segunda parte do incentivo está relacionada ao cumprimento dos critérios anunciados no PHPN. Os critérios foram determinados a partir de dois pressupostos: quais deveriam ser as práticas assistenciais mínimas, ou seja, aquelas que são comprovadamente efetivas e cuja ausência compromete a qualidade da assistência e que são operacionalmente exeqüíveis em curto prazo.

O PHPN estabeleceu um número mínimo de consultas e avaliou a presença ou ausência de determinados recursos de apoio diagnóstico adotando uma estratégia de que, para o país, essas exigências estavam adequadas, e, em cada município, o acréscimo de cuidados dependeria do desempenho local, sendo muito complicado para um país com tantas diferenças e desigualdades regionais contemplar todas as particularidades.

A observação do indicador que monitora a realização de, pelo menos, seis consultas por mulher aponta que ocorre um pequeno incremento no período estudado, mas o percentual se mantém em níveis ainda muito baixos, indicando que, de fato, a realização de seis consultas é ainda um desafio para a assistência. Para esta análise, é preciso levar em conta, em primeiro lugar, que existe um sub-registro das informações referentes ao seguimento das gestantes cadastradas, com desdobramento para todos os outros indicadores. Isso remete à resistência que o profissional de saúde tem para incorporar, na rotina de atendimento, mais um formulário, sem estar sensibilizado e conhecer a razão de estar preenchendo "mais um papel".

Como até dezembro de 2002 o SISPRENATAL só aceitava o cadastramento de mulheres com, no máximo, 120 dias de gravidez, muitas unidades de saúde utilizaram o formulário para registro do acompanhamento da gestante apenas para as cadastradas. Na falta de uma rotina de registro de informações sistemática para todas as gestantes, é provável que exista uma perda significativa de registro de seguimento (consultas, exames) para aquelas que, embora anteriormente cadastradas, não tenham sido assim identificadas no(s) retorno(s). 


\begin{tabular}{|c|c|c|}
\hline Indicadores do processo & Percentual/2001 & Percentual/2002 \\
\hline $\begin{array}{l}\text { 1. Percentual de gestantes que se inscreveram no programa e realizaram a consulta } \\
\text { em relação ao número de nascidos vivos no período. }\end{array}$ & 9,25 & 27,92 \\
\hline $\begin{array}{l}\text { 1.1b. Percentual de gestantes cadastradas no programa no período selecionado em relação ao total } \\
\text { de gestantes cadastradas no banco nacional (Gestantes cadastradas nesta Base: 792.636). }\end{array}$ & 20,80 & 70,14 \\
\hline $\begin{array}{l}\text { 1c. Percentual de gestantes que se inscreveram no programa e realizaram a consulta até } 120 \text { dias } \\
\text { em relação ao número de nascidos vivos no período. }\end{array}$ & 9,12 & 24,59 \\
\hline 2. Percentual de gestantes inscritas que realizaram 6 (seis) consultas de pré-natal. & 19,65 & 22,63 \\
\hline 3. Percentual de gestantes inscritas que realizaram 6 (seis) consultas de pré-natal e a consulta de puerpério. & 6,47 & 9,43 \\
\hline 4. Percentual de gestantes inscritas que realizaram 6 (seis) consultas de pré-natal e todos os exames básicos. & 7,02 & 11,35 \\
\hline $\begin{array}{l}\text { 5. Percentual de gestantes inscritas que realizaram } 6 \text { (seis) consultas de pré-natal, a consulta de puerpério } \\
\text { e todos os exames básicos. }\end{array}$ & 2,78 & 5,59 \\
\hline $\begin{array}{l}\text { 6. Percentual de gestantes inscritas que receberam a } 2 \text { a dose ou a dose de reforço ou a dose imunizante } \\
\text { da vacina antitetânica. }\end{array}$ & 21,79 & 41,01 \\
\hline $\begin{array}{l}\text { 7a. Percentual de gestantes inscritas que realizaram } 6 \text { (seis) consultas de pré-natal, a consulta de puerpério, } \\
\text { todos os exames básicos, a } 2 \underline{a} \text { dose ou dose de reforço ou a dose imunizante da vacina antitetânica. }\end{array}$ & 2,08 & 5,03 \\
\hline $\begin{array}{l}\text { 7. Percentual de gestantes inscritas que realizaram } 6 \text { (seis) consultas de pré-natal, a consulta de puerpério, } \\
\text { todos os exames básicos, o teste anti-HIV, a } 2 \underline{a} \text { dose ou a dose de reforço ou a dose imunizante } \\
\text { da vacina antitetânica. }\end{array}$ & 2,00 & 4,07 \\
\hline 8. Percentual de gestantes inscritas que realizaram o teste anti-HIV. & 41,10 & 35,38 \\
\hline 9. Percentual de gestantes inscritas que realizaram os dois exames VDRL. & 23,10 & 12,11 \\
\hline
\end{tabular}

Outras dificuldades ocorreram, tais como: a perda do número do SISPRENATAL da mulher, as dificuldades para o encaminhamento das fichas para os serviços de digitação nas secretarias e a falta de treinamento dos profissionais do setor de controle e avaliação para a correta digitação e envio dos dados. Além disso, é necessário também reconhecer que a garantia de oferta de, pelo menos, seis consultas durante a gestação requer adequada estrutura e organização dos serviços.

Ainda que se considere a possibilidade de sub-registro de informações referentes ao seguimento das mulheres, é possível afirmar que a realização de exames constitui-se em importante ponto de estrangulamento da assistência no país, e os resultados desse indicador no programa foram reiterados por gestores locais e estaduais em reuniões para monitoramento da implementação da estratégia em todas as regiões do país. Aliás, muitos municípios surpreenderam-se ao estimar as necessidades anuais de exames laboratoriais para atender às gestantes (informação obrigatória para o termo de adesão ao PHPN), pois elas respondiam por quase toda quota de exames disponível para o SUS naquela cidade.

A proposta desenhada para a assistência pré-natal no PHPN estabeleceu a presença de consulta puerperal como critério indispensá- vel no conjunto da assistência. A consulta puerperal é, em primeira análise, a instância do ciclo gravídico-puerperal em que se desvincula a saúde da mãe e do recém-nascido e, portanto, um momento de atenção à saúde da mulher e não da "mãe". Por diferentes razões, essa consulta tem baixíssima freqüência nas UBS, e os percentuais nesse indicador (consulta puerperal e seis consultas de pré-natal) apresentados no SISPRENATAL são menores que $10 \%$, nos dois anos em estudo. Mesmo quando avaliamos a presença da consulta puerperal independentemente da realização dos demais procedimentos, para evitar o viés da acumulação, o maior percentual alcançado é de $19 \%$, quando há uma consulta pré-natal e a consulta puerperal. Teoricamente, os serviços de saúde reconhecem a consulta puerperal como necessária, mas admitem a ausência de estratégias para a sua realização. Outra justificativa para essa baixa freqüência é a alegação mais comum de que “as mulheres não voltam”. Entretanto, como já foi demonstrado, é possível afirmar que "sim, as mulheres voltam aos serviços”, como atestam os dados do Programa Nacional de Imunizações (PNI), mostrando que os recém-nascidos vão aos Postos de Saúde para serem vacinados e geralmente vão com suas mães (http: //www.saude.gov.br, acessado em Fev/2003). De fato, os serviços e profissionais, em geral, 
consideram o parto o "final" do processo e não valorizam esse retorno. Embora seja evidentemente uma questão de falha no planejamento e na execução da assistência, também é, em primeiro lugar, um viés de gênero na percepção dessa atenção.

A realização da vacina antitetânica, uma atividade de absoluto consenso técnico, ainda apresenta percentual aquém do ideal. A primeira hipótese é a ausência de parte dos registros das doses aplicadas, reiterando a necessidade de organização e gestão da assistência. Como a erradicação dos casos de tétano neonatal ainda é uma meta a ser alcançada, esse indicador merece permanente avaliação, principalmente nos municípios em que esse risco é maior.

A questão norteadora para melhorar a qualidade da atenção no pré-natal foi traduzida na realização de todos os critérios instituídos no PHPN por cada mulher. Esta análise, inédita, dos indicadores do SISPRENATAL para todo o país é consistente com resultados das avaliações realizadas em diferentes serviços e municípios sobre essa assistência, que também indicam que apenas um pequeno número de mulheres recebia a assistência adequada. No contexto desta avaliação, da necessidade de melhorar a atenção pré-natal, a estratégia do PHPN em criar um instrumento de avaliação, o

\section{Resumo}

Avalia-se a experiência da implantação do Programa de Humanização no Pré-natal e Nascimento do Ministério da Saúde, no Brasil, a partir dos dados gerados pelo SISPRENATAL/DATASUS. De sua instituição em junho de 2000 até dezembro de 2002, 3.983 municípios aderiram, e 71\% desses (3.183) registraram procedimentos no âmbito do programa, constituindo um banco de dados com 720.871 mulheres. Cerca de 20\% das mulheres realizaram seis ou mais consultas de pré-natal, e cerca da metade delas realizou também a consulta puerperal e os exames obrigatórios em 2002, e $41 \%$ das mulheres foram imunizadas com a vacina antitetânica. A realização de exames para a detecção de HIV foi o dobro em relação à sífilis, nos dois anos em análise. O conjunto das atividades assistenciais recomendado foi recebido apenas por um pequeno percentual de mulheres, $2 \%$ em 2001 e 5\% em 2002. Os baixos percentuais registrados ratificam a necessidade de permanentes avaliações com o objetivo de melhorar a qualidade dessa atenção, garantindo, além de melhores resultados maternos e perinatais, o direito inalienável de cada mulher de gestar e parir com segurança e bem-estar.

Saúde Materno-infantil; Saúde da Mulher; Avaliação de Programas
SISPRENATAL, apresentou-se como um importante instrumento em favor da melhoria da atenção. O sistema possibilitou a informatização e a produção de relatórios municipais que permitem, sinteticamente a qualquer gestor, uma aproximação indispensável da assistência prestada às gestantes em seu território, a partir de informações cuja obtenção demandava um grande esforço até então. O acompanhamento de indicadores é consensualmente apontado como uma medida fundamental para reduzir mortes maternas e perinatais, porque a sinalização dos problemas demanda as medidas para sua resolução. Atualmente, mais de três mil municípios no Brasil podem utilizar esse instrumento na construção de um pré-natal mais adequado. A força fundamental para a concretização das mudanças necessárias está no compromisso político com as premissas filosóficas do SUS e com as mulheres. Esse compromisso, no entanto, deve-se traduzir no trabalho constante de avaliação e readequação da assistência. Programas, estratégias de atenção e monitoramento de resultados são instrumentos auxiliares nesse trabalho. Se não utilizados com essa finalidade, passam a ser percebidos apenas como mais uma tarefa e não acrescentam ganhos à saúde da população.

\section{Colaboradores}

S. J. Serruya foi a responsável pela coleta dos dados para este estudo e pela primeira versão escrita do artigo. S. J. Serruya e J. G. Cecatti escreveram o projeto para este estudo, revisaram e corrigiram os dados, fizeram as análises, construíram as tabelas e revisaram o texto. T. G. Lago contribuiu na idéia original e implantação do programa, revisou e fez sugestões no texto final do artigo. 


\section{Referências}

1. Pitanguy JO. Movimento nacional e internacional de saúde e direitos reprodutivos. In: Giffin K, Costa SH, organizadores. Questões da saúde reprodutiva. Rio de Janeiro: Editora Fiocruz; 1999. p. 19-38.

2. World Health Organization. Making pregnancy safer - a health sector strategy for reducing maternal and e perinatal morbidity and mortality. Geneva: World Health Organization; 2000.

3. Abouzahr C, Wardlaw T. La mortalidad materna al término de una década: hay indicios de progreso? Bull World Health Organ 2001; 79:561-8.

4. Campbell $\mathrm{O}$. What are maternal health policies in developing countries and who drives them? A review of the last half century. In: Brouwere VD, van Lerberghe W, editors. Safe motherhood strategies. A review of the evidence. Antwerp: ITG Press; 2001. p. 415-45.

5. Ghosh MK. Maternal mortality. J Reprod Med 2001; 46:427-33.

6. Goodburn E, Campbell O. Reducing maternal mortality in the developing world: sector-wide approaches may de the key. BMJ 2001; 322:917-20.

7. World Health Organization. Care in normal birth. A practical guide - maternal and newborn health. Geneva: World Health Organization; 1996.

8. World Health Organization/United Nations Population Fund/United Nations Children's Fund/ World Bank. Reduction of maternal mortality: a joint WHO/UNFPA/UNICEF/World Bank statement. Geneva: World Health Organization; 1999.

9. Vaughan JP, Abouzahr C. Reproductive health: widening horizons. Bull World Health Organ 2000; 78:569-73.

10. Pittrof R, Campbell OM, Filippi VG. What is quality in maternity care? An international perspective. Acta Obstet Gynecol Scand 2002; 81:277-83.

11. Davis-Floyd R. The technocratic, humanistic, and holistic paradigms of childbirth. Int J Gynaecol Obstet 2001; 48 Suppl:S33-S52.

12. Osis MJD. PAISM: um marco na abordagem da saúde reprodutiva no Brasil. Cad Saúde Pública 1998; 14 Suppl:25-32.

13. Costa AM. Desenvolvimento e implantação do PAISM no Brasil, In: Giffin K, Costa SH, organizadores. Questões da saúde reprodutiva. Rio de Janeiro: Editora Fiocruz; 1999. p. 319-35.

14. Medici AC. Uma década de SUS (1988-1998): progressos e desafios In: Galvão OL, Diaz J, organizadores. Saúde sexual e reprodutiva no Brasil. São Paulo: Editora Hucitec/Population Council; 1999. p. 104-50.

15. Corrêa S. PAISM: uma história sem fim. Recife: SOS Corpo; 1993.

16. Faúndes A, Cecatti JG, organizadores. Morte materna uma tragédia evitável. Campinas: Editora da Unicamp; 1991.

17. Campbell O, Koblinsky M, Taylor P. Off to a rapid start: appraising maternal mortality and services. Int J Gynaecol Obstet 1995; 48 Suppl:S33-S52.

18. Lumbiganon P. Apropriate technology: antenatal care. Int J Gynecol Obstet 1998; 63 Suppl 1:S91-5.

19. Fenton PM, Tadesse E. Reducing perinatal and maternal mortality in the world: major challenges.
Br J Obstet Gynaecol 2000; 107:831-2.

20. Ronsmans C, Campbell O, McDermott J, Koblinsky M. Questioning the indicators of need for obstetric care. Bull World Health Organ 2002; 80:31724.

21. Bergsjø P. What is the evidence for the role of antenatal care strategies in the reduction of maternal mortality and morbidity? In: Brouwere VD, van Lerberghe W, editors. Safe motherhood strategies. A review of the evidence. Antwerp: ITG Press; 2001. p. 35-54.

22. Silveira DS, Santos IS, Costa JSD. Atenção pré-natal na rede básica: uma avaliação da estrutura e do processo. Cad Saúde Pública 2001; 17:131-9.

23. Victora CG, Barros FC. Infant mortality due to perinatal causes in Brazil: trends, regional patterns and possible interventions. São Paulo Med J 2001; 119:33-43.

24. Trevisan MR, Delorenzi DR, Araújo NM, Êsber K. Perfil da assistência pré-natal entre usuárias do sistema único de saúde em Caxias do Sul. Rev Bras Ginecol Obstet 2002; 24:293-9.

25. Villar J, Garcia P, Walker G. Routine antenatal care. Curr Opin Obstet Gynecol 1993; 5:688-93.

26. Villar J, Khan-Neelofur D. Routine antenatal care for low-risk pregnancy. How often and by whom should antenatal care be provided? (Cochrane Review). In: The Cochrane Library, Issue 3, 1997. Oxford: Update Software.

27. Bergsjø P, Villar J. Scientific basis for the content of routine antenatal care. Acta Obstet Gynecol Scand 1997; 76:15-25.

28. Carroli G, Rooney C, Villar J. How effective is antenatal care in preventing maternal mortality and serious morbidity? An overview of the evidence. Paediatr Perinat Epidemiol 2001; 15 Suppl 1:1-42.

29. Pinol A, Bergel E, Chaisiri K, Diaz E, Gandeh M. Managing data for a randomised controlled clinical trial: experience from the WHO Antenaral Care Trial. Paediatr Perinat Epidemiol 1998; 12:142-55.

30. Villar J, Ba'Aqeel H, Piaggio G, Lumbiganon P, Belizán J, Farnot U, et al. WHO antenatal care randomised trial for the evaluation of a new model of routine antenatal care. Lancet 2001; 357:1551-64.

31. World Health Organization. WHO antenatal care randomized trial: manual for the implementation of the new model. Geneva: World Health Organization; 2002.

32. Puccini RF, Pedroso GC, Silva EM, Araújo NS, Silva NN. Eqüidade na atenção pré-natal e ao parto em área da Região Metropolitana de São Paulo, 1996. Cad Saúde Pública 2003; 19:35-45.

33. Organização Pan-Americana da Saúde. Rede interagencial de informações para a saúde. Indicadores básicos para a saúde no Brasil: conceitos e aplicações. Brasília: Organização Pan-Americana da Saúde; 2002.

34. Tanaka ACA. Maternidade dilema entre nascimento e morte. São Paulo: Editora Hucitec/Rio de Janeiro: ABRASCO; 1995.

Recebido em 18/Nov/2003

Versão final reapresentada em 16/Mar/2004

Aprovado em 04/Mai/2004 